\title{
Reduced Free Products of Completely Positive Maps and Entropy for Free Product of Automorphisms
}

By

\author{
Marie CHODA*
}

\begin{abstract}
The reduced free product of unital completely positive maps is defined. An invariant state on a $C^{*}$-algebra for an automorphism (based on the free shift) is the composition of a state of a subalgebra with the reduced free product of expectations. Entropies for the reduced free product and the tensor product of an automorphism $\gamma$ with the free shift coincide with the entropy of $\gamma$.
\end{abstract}

\section{§1. Introduction}

The reduced free product $(A, \phi)=\left(* A_{i}, * \phi_{i}\right)_{i \in I}$ of unital $C^{*}$-algebras $\left\{A_{i}\right\}_{i \in I}$ with respect to their states $\left\{\phi_{i}\right\}_{i \in I}$ is defined by Avitzour in [A] and Voiculescu in [V] independently.

For a unital $C^{*}$-algebra $B_{i},(i \in I)$ with a state $\psi_{i}$, let $T_{i}$ be a unital completely positive linear map from $A_{i}$ to $B_{i}$ with $\psi_{i} \cdot T_{i}=\phi_{i}$. Put $(B, \psi)=\left(* B_{i}, * \psi_{i}\right)_{i \in I}$. In $\S 2$, we define the reduced free product $T=*_{i \in I} T_{i}$, which is a unital completely positive map from $A$ to $B$ with $\psi \cdot T=\phi$. If $B_{i}$ is a $C^{*}$-subalgebra of $A_{i}$ and $T_{i}$ is a conditional expectation $E_{i}$ from $A_{i}$ onto $B_{i}$, then $*_{i \in I} E_{i}$ is still a conditional expectation from $A$ onto $B$. If $B_{i}=A_{i}$ and $T_{i}$ is an automorphism $\theta_{i}$, then $*_{i \in I} \theta_{i}$ is an automorphism of $A$.

If the index set $I$ is the integers $\mathbb{Z}$ and $A_{i}=A_{0}$ for all $i \in \mathbb{Z}$, then we have the automorphism $\alpha$ of $A$ which comes from the shift: $n \in \mathbb{Z} \rightarrow n+1$. The $\alpha$ is called the free shift [S]. In $\S 3$, using the reduced free product of some conditional expectations, we show an extended version of Avitzour's uniquely ergodic theorem [A: 4.1 Proposition] for the free shift.

Sauvageot and Thouvenot [ST] give a definition of entropy $H_{\rho}(\gamma)$ for a $\rho$-invariant automorphism $\gamma$ of a unital $C^{*}$-algebra $C$ with a state $\rho$. Their

Communicated by H. Araki, October 19, 1995.

1991 Mathematical Subject Classification: 46L55, 46L35, 46L40

* Department of Mathematics, Osaka Kyoiku University, Asahigaoka, Kashiwara 582, Japan 
entropy coincides with that of Connes, Narnhofer and Thirring [CNT] if $C$ is nuclear. In $\S 4$, as an application of the result in $\S 3$, we show that the free shift $\alpha$ does not change Sauvageot-Thouvenot entropy for the reduced free product and the tensor product:

$$
H_{\phi * \rho}(\alpha * \gamma)=H_{\rho}(\gamma)=H_{\phi \otimes \rho}(\alpha \otimes \gamma)
$$

for every $\rho$ invariant automorphism $\gamma$ of $C$. Applying this to the identity automorphism of $C$, we have $H_{\phi}(\alpha)=0([\mathrm{~S}:$ Remark 2]).

\section{§2. Reduced Free Products of Completely Positive Maps}

In this section, we define the reduced free product of unital completely positive linear maps. First, to fix notations, we recall definition of reduced free product of unital $C^{*}$-algebras. Let $I$ be an index set. For each $i \in I$ let $A_{i}$ be a unital $C^{*}$-algebra and $\Phi_{i}: A_{i} \rightarrow B\left(H_{i}\right)$ be a ${ }^{*}$-representation on the Hilbert space $H_{i}$ with the distinguished unit vector $\xi_{i}$. The free product Hilbert space $\left(* H_{i}, * \xi_{i}\right)_{i \in I}$ is $(H, \xi)$ with

$$
H=\mathbb{C} \xi \oplus \underset{n \geq 1}{\bigoplus}\left(\underset{i_{1} \neq \cdots \neq i_{n}}{\bigoplus} H_{i_{1}}^{\circ} \otimes \cdots \otimes H_{i_{n}}^{\circ}\right) .
$$

Here $H_{i}^{\circ}=H_{i} \ominus \mathbb{C} \xi_{i}$. Put

$$
H(i)=\mathbb{C} \xi \oplus \underset{n \geq 1}{\oplus}\left(\underset{i \neq i_{1} \neq i_{2} \neq \cdots i_{n}}{\bigoplus} H_{i_{1}}^{\circ} \otimes \cdots \otimes H_{i_{n}}^{\circ}\right) .
$$

The unitary operator $V_{i}: H_{i} \otimes H(i) \rightarrow H$ is defined by

$$
\begin{aligned}
& \xi_{i} \otimes \xi \rightarrow \xi \\
& H_{i}^{\circ} \otimes \xi \rightarrow H_{i}^{\circ} \text { by } \eta \otimes \xi \rightarrow \eta \\
& \xi_{i} \otimes\left(H_{i_{1}}^{\circ} \otimes \cdots \otimes H_{i_{n}}^{\circ}\right) \rightarrow H_{i_{1}}^{\circ} \otimes \cdots \otimes H_{i_{n}}^{\circ} \text { by } \xi_{i} \otimes \eta \rightarrow \eta,\left(i_{1} \neq i\right) \\
& H_{i}^{\circ} \otimes\left(H_{i_{1}}^{\circ} \otimes \cdots \otimes H_{i_{n}}^{\circ}\right) \rightarrow H_{i}^{\circ} \otimes H_{i_{1}}^{\circ} \otimes \cdots \otimes H_{i_{n}}^{\circ} \text { by } \psi \otimes \eta \rightarrow \psi \otimes \eta,\left(i_{1} \neq i\right) .
\end{aligned}
$$

The representation $\lambda_{i}: A_{i} \rightarrow B(H)$ is defined by

$$
\lambda_{i}(a)=V_{i}\left(\Phi_{i}(a) \otimes 1_{H(i)}\right) V_{i}^{*}, \quad\left(a \in A_{i}\right) .
$$

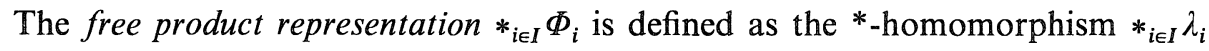
from the enveloping $C^{*}$-algebra $*_{i \in I} A_{i}$ of the ${ }^{*}$-algebra free product of the $\left\{A_{i}\right\}_{i \in I}$ to $B(H)$ using the universal property of the free product ([VDN]).

Now, let $A_{i}$ be a unital $C^{*}$-algebra and $\phi_{i}$ a state on $A_{i}$. We denote the GNS representation of $A_{i},(i \in I)$ with respect to $\phi_{i}$ by $\left(\pi_{i}, H_{i}, \xi_{i}\right)$. Then we have the above representation $\lambda_{i}: A_{i} \rightarrow B(H)$ coming from $\pi_{i}$. The reduced free product $(A, \phi)=\left(* A_{i}, * \phi_{i}\right)_{i \in I}$ is the $C^{*}$-algebra $A$ on $H$ generated by 
$\bigcup_{i \in I} \lambda_{i}\left(A_{i}\right)$ and the vector state $\phi: \phi(a)=\langle a \xi, \xi\rangle, a \in A$ ([V]).

Usually, for $a \in A_{i}$, we denote $\pi_{i}(a)$ by $a$, and also $\lambda_{i}\left(\pi_{i}(a)\right)$ by $a$, for simplicity of notation. For each $i \in I$, let

$$
\stackrel{\circ}{A}_{i}=\left\{a \in A_{i}: \phi_{i}(a)=0\right\}
$$

and

$$
\operatorname{red}(A)=\left\{a_{i_{1}} \cdots a_{i_{n}}: a_{i_{k}} \in \stackrel{\circ}{A}_{i_{k}}, i_{1} \neq \cdots \neq i_{n}\right\} .
$$

We call an $a \in \operatorname{red}(A)$ a reduced word in $A$. Then $\mathbb{C} 1+$ linear span $\operatorname{red}(A)$ is dense in $A$ and $\phi(a)=0$ for a reduced word $a$ in A.

Proposition 2.1. Let $A_{i}$ and $B_{i}$ be unital $C^{*}$-algebras with states $\phi_{i}$ and $\psi_{i},(i \in I)$ respectively and let $T_{i}$ be a unital completely positive map of $A_{i}$ to $B_{i}$ with $\psi_{i} \cdot T_{i}=\phi_{i}$. Let $(A, \phi)=\left(* A_{i}, * \phi_{i}\right)_{i \in I}$ and $(B, \psi)=\left(* B_{i}, * \psi_{i}\right)_{i \in I}$. Then there exists a unital completely positive map $T$ of $A$ to $B$ which satisfies

$$
\psi \cdot T=\phi \quad \text { and } \quad T(a)=T_{i}(a), \quad\left(a \in A_{i}\right)
$$

and $T(\operatorname{red}(A)) \subset \operatorname{red}(B)$, more precisely

$$
T\left(a_{i_{1}} a_{i_{2}} \cdots a_{i_{n}}\right)=T_{i_{1}}\left(a_{i_{1}}\right) T_{i_{2}}\left(a_{i_{2}}\right) \cdots T_{i_{n}}\left(a_{i_{n}}\right), \quad\left(i_{k} \neq i_{k+1}, a_{i_{j}} \in \dot{A}_{i_{j}}\right) .
$$

Proof. Let $(H, \xi)$ be as above and $\left(\beta_{i}, K_{i}, \eta_{i}\right)$ be the GNS representation of $B_{i}$ by $\psi_{i}$. Then by Stinespring's dilation theorem, there exist a Hilbert space $L_{i}$, a ${ }^{*}$-representation $\Phi_{i}: A_{i} \rightarrow B\left(L_{i}\right)$ and an isometry $W_{i}: K_{i} \rightarrow L_{i}$ such that

$$
T_{i}(a)=W_{i}^{*} \Phi_{i}(a) W_{i}, \quad\left(a \in A_{i}\right) .
$$

Put

$$
(K, \eta)=\left(* K_{i}, * \eta_{i}\right)_{i \in I}, \zeta_{i}=W_{i} \eta_{i},(i \in I) \text { and }(L, \zeta)=\left(* L_{i}, * \zeta_{i}\right)_{i \in I} .
$$

Then we have an isometry $W: K \rightarrow L$ defined by

$$
\left\{\begin{aligned}
W \eta & =\zeta \\
W\left(v_{i_{1}} \otimes \cdots \otimes v_{i_{n}}\right) & =W_{i_{1}} v_{i_{1}} \otimes \cdots \otimes W_{i_{n}} v_{i_{n}},\left(i_{i} \neq i_{j+1}, v_{i_{j}} \in K_{i_{j}}^{\circ}\right) .
\end{aligned}\right.
$$

Let $\Phi$ be the free product representation $*_{i \in I} \Phi_{i}$. We define $T$ by

$$
T(a)=W^{*} \Phi(a) W, \quad(a \in A) .
$$

It is obvious that $T: A \rightarrow B(K)$ is a unital completely positive map.

For $a \in \stackrel{\circ}{A}_{i}$,

$$
\left\langle\Phi_{i}(a) \zeta_{i}, \zeta_{i}\right\rangle=\left\langle T_{i}(a) \eta_{i}, \eta_{i}\right\rangle=\psi_{i}\left(T_{i}(a)\right)=\phi_{i}(a)=0 .
$$

Hence $T_{i}(a) \eta_{i} \in K_{i}^{\circ}, \Phi_{i}(a) \zeta_{i} \in L_{i}^{\circ}$ for $a \in \dot{A}_{i}$ and $T_{i}\left(\dot{A}_{i}\right) \subset \dot{B}_{i}$. For a while we denote Voiculescu's unitary $V_{i}: K_{i} \otimes K(i) \rightarrow K$ by $V_{i}{ }^{B}$ and the representation 
$\lambda_{i}$ of $B_{i}$ into $B$ by $\lambda_{i}^{B}$. The notations of unitary representation $V_{i}$ and the representation $\lambda_{i}$ are used for the free product representation $*_{i \in I} \Phi_{i}$. First we remark

$$
\begin{aligned}
& T_{i_{1}}\left(a_{i_{1}}\right) \eta_{i_{1}} \otimes \cdots \otimes T_{i_{n}}\left(a_{i_{n}}\right) \eta_{i_{n}} \\
& \quad=\lambda_{i_{1}}^{B}\left(T_{i_{1}}\left(a_{i_{1}}\right)\right) \cdots \lambda_{i_{n}}^{B}\left(T_{i_{n}}\left(a_{i_{n}}\right)\right) \eta, \quad\left(i_{k} \neq i_{k+1}, a_{i_{j}} \in \AA_{i_{j}}\right) .
\end{aligned}
$$

Hence

$$
T\left(a_{i_{1}} a_{i_{2}} \cdots a_{i_{n}}\right) \eta=\lambda_{i_{1}}^{B}\left(T_{i_{1}}\left(a_{i_{1}}\right)\right) \cdots \lambda_{i_{n}}^{B}\left(T_{i_{n}}\left(a_{i_{n}}\right)\right) \eta .
$$

Next we consider a vector $v=v_{j_{1}} \otimes \cdots \otimes v_{j_{m}},\left(j_{k} \neq j_{k+1}, v_{j_{k}} \in K_{j_{k}}^{\circ}\right)$.

If $j_{1} \neq i_{n}$. We have

$$
\lambda_{i_{1}}^{B}\left(T_{i_{1}}\left(a_{i_{1}}\right)\right) \cdots \lambda_{i_{n}}^{B}\left(T_{i_{n}}\left(a_{i_{n}}\right)\right) v=T_{i_{1}}\left(a_{i_{1}}\right) \eta_{i_{1}} \otimes \cdots \otimes T_{i_{n}}\left(a_{i_{n}}\right) \eta_{i_{n}} \otimes v .
$$

Hence we have

$$
T\left(a_{i_{1}} a_{i_{2}} \cdots a_{i_{n}}\right) v=\lambda_{i_{1}}^{B}\left(T_{i_{1}}\left(a_{i_{1}}\right)\right) \cdots \lambda_{i_{n}}^{B}\left(T_{i_{n}}\left(a_{i_{n}}\right)\right) v .
$$

Assume $j_{1}=i_{n}$ and $m=1$ (hence $v=v_{i_{n}}$ ). We decompose $T_{i_{n}}\left(a_{i_{n}}\right) v_{i_{n}}$ into the direct sum:

$$
T_{i_{n}}\left(a_{i_{n}}\right) v_{i_{n}}=c_{i_{n}} \eta_{i_{n}}+\left[T_{i_{n}}\left(a_{i_{n}}\right) v_{i_{n}}-c_{i_{n}} \eta_{i_{n}}\right] \in \mathbb{C} \eta_{i_{n}} \oplus K_{i_{n}}^{\circ},
$$

then $\Phi_{i_{n}}\left(a_{i_{n}}\right) W_{i_{n}} v_{i_{n}}=c_{i_{n}} \zeta_{i_{n}}+\left[\Phi_{i_{n}}\left(a_{i_{n}}\right) W_{i_{n}} v_{i_{n}}-c_{i_{n}} \zeta_{i_{n}}\right] \in \mathbb{C} \zeta_{i_{n}} \oplus L_{i_{n}}^{\circ}$, where

$$
c_{i}=\left\langle\Phi_{i}(a) W_{i} v_{i}, \zeta_{i}\right\rangle=\left\langle T_{i_{n}}\left(a_{i_{n}}\right) v_{i_{n}}, \eta_{i_{n}}\right\rangle \text {. }
$$

Put

$$
v_{i_{n}}^{\prime}=\Phi_{i_{n}}\left(a_{i_{n}}\right) W_{i_{n}} v_{i_{n}}-c_{i_{n}} \zeta_{i_{n}} .
$$

Then $T_{i_{n}}\left(a_{i_{n}}\right) v_{i_{n}}=c_{i_{n}} \eta_{i_{n}}+W_{i_{n}}^{*} v_{i_{n}}^{\prime}$. Since by the above discussion,

$$
\lambda_{i_{1}}^{B}\left(T_{i_{1}}\left(a_{i_{1}}\right)\right) \cdots \lambda_{i_{n}}^{B}\left(T_{i_{n}}\left(a_{i_{n}}\right)\right) v_{i_{n}}=c_{i_{n}} T\left(a_{i_{1}} \cdots a_{i_{n-1}}\right) \eta+T\left(a_{i_{1}} \cdots a_{i_{n-1}}\right) \eta \otimes W_{i_{n}}^{*} v_{i_{n}}^{\prime},
$$

we have

$$
\lambda_{i_{1}}^{B}\left(T_{i_{1}}\left(a_{i_{1}}\right)\right) \cdots \lambda_{i_{n}}^{B}\left(T_{i_{n}}\left(a_{i_{n}}\right)\right) \nu=c_{i_{n}} T\left(a_{i_{1}} \cdots a_{i_{n-1}}\right) \eta+T\left(a_{i_{1}} \cdots a_{i_{n-1}}\right) \eta \otimes W_{i_{n}}^{*} v_{i_{n}}^{\prime}
$$

which implies

$$
T\left(a_{i_{1}} a_{i_{2}} \cdots a_{i_{n}}\right) v=\lambda_{i_{1}}^{B}\left(T_{i_{1}}\left(a_{i_{1}}\right)\right) \cdots \lambda_{i_{n}}^{B}\left(T_{i_{n}}\left(a_{i_{n}}\right)\right) v .
$$

If $j_{1}=i_{n}$ and $m \geq 2$. We use again the above direct decomposition of $\Phi_{i_{n}}\left(a_{i_{n}}\right) v_{i_{n}}$ and $T_{i_{n}}\left(a_{i_{n}}\right) v_{i_{n}}$. Put

$$
v(2)=v_{j_{2}} \otimes \cdots \otimes v_{j_{m}},
$$

then we have 


$$
\begin{aligned}
T\left(a_{i_{1}} a_{i_{2}} \cdots a_{i_{n}}\right) v=c_{i_{n}} W^{*}\left(\Phi\left(a_{i_{1}}\right) \cdots\right. & \left.\Phi\left(a_{i_{n-1}}\right) W v(2)\right) \\
& +W^{*}\left(\Phi\left(a_{i_{1}}\right) \cdots \Phi\left(a_{i_{n-1}}\right)\left(v_{i_{n}}^{\prime} \otimes W v(2)\right)\right) .
\end{aligned}
$$

We put

$$
(*)=c_{i_{n}} W^{*}\left(\Phi\left(a_{i_{1}}\right) \cdots \Phi\left(a_{i_{n-1}}\right) W v(2)\right)
$$

and

$$
(* *)=W^{*}\left(\Phi\left(a_{i_{1}}\right) \cdots \Phi\left(a_{i_{n-1}}\right)\left(v_{i_{n}}^{\prime} \otimes W v(2)\right)\right) .
$$

Then $(* *)$ is reduced to the first case.

Assume that $n=1$. Since $W^{*} v_{i_{1}}^{\prime}=\left[T_{i_{1}}\left(a_{i_{1}}\right) v_{i_{1}}-c_{i_{1}} \eta_{i_{1}}\right]$, we have

$$
\lambda_{i_{1}}^{B}\left(T_{i_{1}}\left(a_{i_{1}}\right)\right) v=c_{i_{1}} v(2)+W^{*} v_{i_{1}}^{\prime} \otimes v(2),
$$

so that, by (2.1.4)

$$
T\left(a_{i_{1}}\right) v=\lambda_{i_{1}}^{B}\left(T_{i_{1}}\left(a_{i_{1}}\right)\right) v .
$$

Assume that $n \geq 2$. We compute (*) in the two cases $i_{n-1}=j_{2}$ and $i_{n-1} \neq j_{2}$, and iterate the above discussions. Then

$$
T\left(a_{i_{1}} a_{i_{2}} \cdots a_{i_{n}}\right) v=\lambda_{i_{1}}^{B}\left(T_{i_{1}}\left(a_{i_{1}}\right)\right) \cdots \lambda_{i_{n}}^{B}\left(T_{i_{n}}\left(a_{i_{n}}\right)\right) v .
$$

As we mentioned before, we denote $\lambda_{i}^{B}\left(T_{i}\left(a_{i}\right)\right)$ simply by $T_{i}\left(a_{i}\right)$ and we have

$$
T\left(a_{i_{1}} a_{i_{2}} \cdots a_{i_{n}}\right)=T_{i_{1}}\left(a_{i_{1}}\right) T_{i_{2}}\left(a_{i_{2}}\right) \cdots T_{i_{n}}\left(a_{i_{n}}\right), \quad\left(i_{k} \neq i_{k+1}, a_{i_{j}} \in \dot{A}_{i_{j}}\right) .
$$

This relation implies that $T(\operatorname{red}(A)) \subset \operatorname{red}(B)$. If $a \in A_{i}$, then $T(a)=T\left(\phi_{i}(a) 1\right.$ $+[a-\phi(a) 1])=\phi_{i}(a) 1+T_{i}\left(a-\phi_{i}(a) 1\right)=T_{i}(a)$. Since $\mathbb{C} 1+$ linear span $\operatorname{red}(A)$ and $\mathbb{C} 1+$ linear span $\operatorname{red}(B)$ are dense in $A$ and $B$ respectively, we have $T(A) \subset B$.

Let $a \in \operatorname{red}(A)$, then $T(a) \in \operatorname{red}(B)$ so that

$$
\psi \cdot T(a)=0=\phi(a), \quad(a \in \operatorname{red}(A)) .
$$

It implies $\psi \cdot T(a)=\phi(a)$ for all $a \in A$.

Definition. We call the $T$ in Proposition 2.1 the reduced free product of completely positive maps $\left\{T_{i}\right\}_{i \in I}$ and denote it by $*_{i \in I} T_{i}$ or as $T_{1} * \cdots * T_{n}$ for the reduced free product for a finite index set I.

It is pointed out by Enomoto and Takehana that in [B: theorem 3.1] Boca shows the completely positivety of the free product (not reduced free product) of unital completely positive linear maps.

It is well known that conditional expectations and *automorphisms are typical examples of unital completely positive maps. Applying Proposition 2.1 to these completely positive maps, we have the following two Corollaries: 
Corollary 2.2. Let $(A, \phi)$ be as in Proposition 2.1. If $E_{i}$ is a conditional expectation of $A_{i}$ onto a unital $C^{*}$-subalgebra $B_{i}$ of $A_{i}$ with $\phi_{i} \cdot E_{i}=\phi_{i}$ then the reduced free product $*_{i \in I} E_{i}$ is a conditional expectation $E$ of $A$ onto $B=\left(* B_{i}, * \phi_{i}\right)_{i \in I}$ with $\phi=\phi \cdot E$,

$$
E\left(a_{i_{1}} a_{i_{2}} \cdots a_{i_{n}}\right)=E_{i_{1}}\left(a_{i_{1}}\right) E_{i_{2}}\left(a_{i_{2}}\right) \cdots E_{i_{n}}\left(a_{i_{n}}\right), \quad\left(i_{k} \neq i_{k+1}, a_{i_{J}} \in \dot{A}_{i_{J}}\right)
$$

and

$$
E(a)=E_{i}(a), \quad\left(a \in A_{i}\right) .
$$

Proof. By Proposition 2.1, we only need to prove the conditional expectation property

$$
E(a b)=E(a) b, \quad(a \in A, b \in B) .
$$

This follows from the argument on [CE: page 166], because $E$ is completely positive and satisfies that $E(b)=b,(b \in B)$.

Corollary 2.3. Let $\alpha_{i}$ be $a^{*}$-automorphism of $A_{i}$ with $\phi_{i} \cdot \alpha_{i}=\phi_{i}$. Then the reduced free product $*_{i \in I} \alpha_{i}$ is a ${ }^{*}$-automorphism $\alpha$ of the reduced free product $(A, \phi)$ with $\phi \cdot \alpha=\phi$ and $\alpha\left(a_{i}\right)=\alpha_{i}\left(a_{i}\right),\left(a_{i} \in A_{i}\right)$.

Proof. Since $*_{i \in I} \alpha_{i}$ maps a reduced word in $A$ to a reduced word in $A$, it is clear that $*_{i \in I} \alpha_{i}$ is an automorphism if every $\alpha_{i}$ is an automorphism.

\section{§3. Invariant States on Reduced Free Products}

Let $A_{0}$ be a unital $C^{*}$-algebra with a state $\phi_{0}$. Put $A_{i}=A_{0}, \phi_{i}=\phi_{0}$

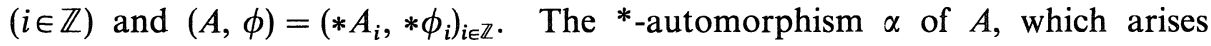
from the shift: $n \rightarrow n+1$ on $\mathbb{Z}$, is called the free shift on $(A, \phi)=\left(* A_{i}, * \phi_{i}\right)_{i \in \mathbb{Z}}$.

We denote by $E_{\phi}$ the conditional expectation of $A$ onto $\mathbb{C} 1$ conditioned by $\phi$, that is, $E_{\phi}(a)=\phi(a) 1$. Then the reduced free product $E_{\phi} * i d_{C}$ is a conditional expectation of $A * C$ onto $C$ by Corolary 2.2 .

The following theorem is an extended version of [A: 4.1 Proposition] and we prove it by an analogous method as the proof of Theorem 3 in early version of $[\mathrm{S}]$.

Theorem 3.1. Let $B$ and $C$ be unital $C^{*}$-algebras with states $\mu$ and $\rho$ respectively. Let $\alpha$ be the free shift on $A, \gamma$ a $\rho$-invariant automorphism on $C$ and $\beta$ a $\mu$-invariant automorphism of $B$. For the reduced free product $(A * C, \phi * \rho)$ if $\psi$ is a state of $(A * C) \otimes B$ with $\psi \cdot((\alpha * \gamma) \otimes \beta)=\psi$ and $\psi_{1}$ is the restriction of $\psi$ to $C \otimes B$, then

$$
\psi=\psi_{1} \cdot F
$$

where $F$ is the conditional expectation of $(A * C) \otimes B$ onto $C \otimes B$ defined by 


$$
F=\left(E_{\phi} * i d_{C}\right) \otimes i d_{B}
$$

Proof. Put $D_{1}=A$ and $D_{2}=C$. Let $S$ be the set of reduced words in $A * C$ which are not contained in $C$ and each component in $A$ is a reduced word in $A$ too. That is, $y \in S$ if and only if $y$ satisfies the following two conditions :

$$
y=y_{i_{1}, 1} y_{i_{2}, 2} \cdots y_{i_{n}, n} \notin C, \quad\left(i_{j} \in\{1,2\}, i_{j} \neq i_{j+1}, y_{i_{j}, j} \in \stackrel{\circ}{D}_{i_{j}}\right)
$$

and if $y_{i_{j}, j} \in \AA$ then $a_{j}=y_{i_{j}, j}$ has the form

$$
a_{j}=a_{j_{1}} a_{j_{2}} \cdots a_{j_{n(J)}} \text { for } \quad a_{j_{k}} \in{\stackrel{\circ}{i\left(j_{k}\right)}}, \quad\left(1 \leq k \leq n(j), i\left(j_{k}\right) \neq i\left(j_{k+1}\right)\right) .
$$

Since $\mathbb{C} 1+$ linear span $\operatorname{red}(A)$ is dense in $A$ and $\mathbb{C} 1+$ linear span $\operatorname{red}(A * C)$ is dense in $A * C$, it is enough to show that $\psi(x)=\psi \cdot F(x)$ for $x=\sum_{k=1}^{n} x_{k} \otimes b_{k}$ $+\sum_{i=1}^{m} c_{i} \otimes b_{i}^{\prime},\left(x_{k} \in S, b_{k}, b_{i}^{\prime} \in B, c_{i} \in \stackrel{\circ}{C}\right)$. Since $F$ is a conditional expectation onto $C \otimes B$ and transforms a reduced word to a reduced word,

$$
F\left(x_{k} \otimes b_{k}\right)=E_{\phi} * i d_{C}\left(x_{k}\right) \otimes b_{k}=0, \quad\left(x_{k} \in S\right) .
$$

Hence we need only to show $\psi(x)=0$ for a self adjoint $x$ which has the form

$$
x=\sum_{i=1}^{n} x_{i} \otimes b_{i}, \quad\left(x_{i} \in S, b_{i} \in B\right) .
$$

Let $\left(H_{i}, \xi_{i}\right)$ and $(L, \zeta)$ be the GNS representation spaces and the vectors of $A_{i}$ and $C$ by $\phi_{i}$ and $\rho$, respectively. Let $(H, \xi)=\left(* H_{i} * \xi_{i}\right)_{i \in \mathbb{Z}}$ and $(K, \eta)=(H * L, \xi * \zeta)$.

We need the following lemma:

Lemma 3.2. If $x$ has the form (3.1.2), then there exists a subspace $K_{x}$ of $K$ such that

$$
x_{i}\left(K_{x}^{\perp}\right) \subset K_{x}, \quad(1 \leq i \leq n) .
$$

Proof. For an $y \in S$, let $J(y)$ be the set of indices $i\left(j_{k}\right)$ such that some $a_{j_{k}} \in{\stackrel{\circ}{A\left(j_{k}\right)}}$ appears in the decomposition (3.1.1) of $a_{j}$ and the $a_{j} \in \AA$ is a component of a reduced word $y$. Put

$$
I(x)=\bigcup_{k=1}^{n} J\left(x_{k}\right)
$$

Let

$$
H_{x}=\bigoplus_{m \geq 1}\left(\underset{\substack{i_{1} \neq \ldots \neq i_{m} \\ i_{1} \in I(x)}}{\bigoplus} \stackrel{\circ}{H}_{i_{1}} \otimes \cdots \otimes \stackrel{\circ}{H}_{i_{m}}\right)
$$

and 


$$
H_{x}^{\prime}=\bigoplus_{m \geq 1}\left(\underset{\substack{i_{1} \neq \ldots \neq i_{m} \\ i_{1} \notin I(x)}}{\bigoplus} \stackrel{\circ}{H}_{i_{1}} \otimes \cdots \otimes \stackrel{\circ}{H}_{i_{m}}\right) .
$$

Put $K_{1}=\stackrel{\circ}{H}$ and $K_{2}=\stackrel{\circ}{L}$. Let

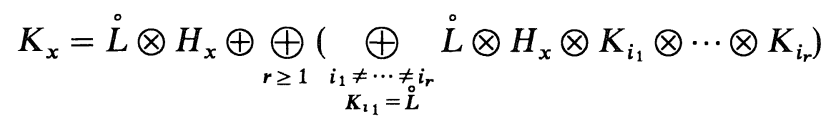

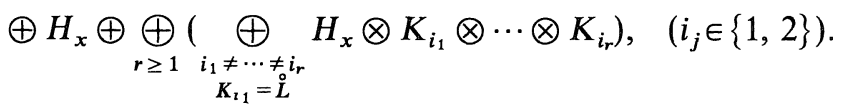

Then

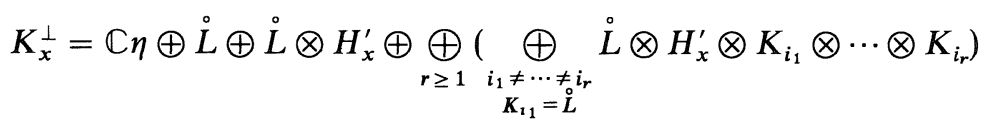

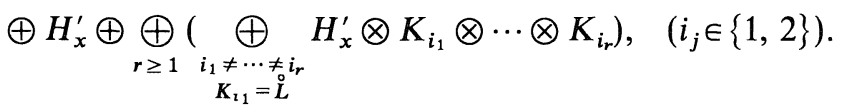

To show $x_{i}\left(K_{x}^{\perp}\right) \subset K_{x}$, we divide the form of $x_{i o}$ (put $=y$ for simplicity of notation) into the following two cases, where $c_{j} \in \stackrel{\circ}{C}$ and $a_{j}$ has the given form (3.1.1).

Case 1: $y=a_{1} c_{1} \cdots a_{n},(n \geq 1)$ or $y=c_{1} a_{1} \cdots a_{n},(n \geq 1)$. Since $a_{j}$ has the form $a_{j}=a_{j_{1}} a_{j_{2}} \cdots a_{j_{n(j)}}$ and $a_{j_{l}} \in \AA_{i\left(j_{l}\right)},\left(1 \leq l \leq n(j), i\left(j_{k}\right) \neq i\left(j_{k+1}\right)\right), a_{j} \xi=$ $a_{j_{1}} \xi_{j_{1}} \otimes \cdots \otimes a_{j_{n(J)}} \xi_{j_{n(J)}} \in H_{x}$. Hence $y \eta=a_{1} \xi \otimes c_{1} \xi \otimes \cdots a_{n} \xi \in K_{x}$. Similarly for the second form. Let $v=v_{i_{1}} \otimes \cdots \otimes v_{i_{r}} \in K_{i_{1}} \otimes \cdots \otimes K_{i_{r}},\left(i_{j} \neq i_{j+1}, v_{i_{j}} \in K_{i_{j}}\right)$, which satisfies the conditions for the components in $K_{x}$. Then $y v=y \eta \otimes$ $v \in K_{x}$.

Case 2: $\quad y=a_{1} \cdots a_{n-1} c_{n},(n \geq 1)$ or $y=c_{1} a_{1} \cdots a_{n-1} c_{n},(n \geq 2)$. For the vector $\eta$ and $v=v_{i_{1}} \otimes \cdots \otimes v_{i_{r}} \in K_{i_{1}} \otimes \cdots \otimes K_{i_{r}},\left(i_{1} \neq \cdots \neq i_{r}, v_{i_{j}} \in K_{i_{j}}, K_{i_{1}}=H_{x}^{\prime}\right)$, similar discussion to the Case 1 implies $y \eta \in K_{x}$ and $y v \in K_{x}$. Assume $v_{i_{1}} \in \stackrel{\circ}{L}$. Put $c_{n} v=s \zeta+\left[c_{n} v-s \zeta\right]$, where $s=\left\langle c_{n} v, \zeta\right\rangle$. Put $y=z c_{n}$. Then $z \eta$ has the form $a_{1} \xi \otimes \cdots \otimes a_{n-1} \xi$ or $c_{1} \zeta \otimes \cdots \otimes a_{n-1} \xi$. Hence $y v=s z \eta+$ $z \eta \otimes\left[c_{n} v-s \zeta\right] \in K_{x}$.

Continuation of the Proof of Theorem 3.1. Let $Y$ be the GNS representation space of $B$ by $\mu$. Let

$$
Z_{x}=K_{x} \otimes Y
$$

Then $Z_{x}^{\perp}=K_{x}^{\perp} \otimes Y$ and by Lemma $3.2 x\left(Z_{x}^{\perp}\right) \subset Z_{x}$. Since $\alpha$ is the free shift there exists integers $0=n_{1}<n_{2}<\cdots<n_{20}$ such that if $\alpha_{0}$ denotes the shift on $\mathbb{Z}$ then the sets $\alpha_{0}^{n_{2}}(I(x)),(1 \leq i \leq 20)$ are all disjoint. Put 


$$
x(i)=((\alpha * \gamma) \otimes \beta)^{n_{2}}(x) \quad \text { and } \quad \hat{x}=(1 / 20) \sum_{k=i}^{20} x(i)
$$

Since $\alpha$ maps a reduced word in $A$ to a reduced word in $A$, by a property of the reduced free product $\alpha * \gamma(x(i))$ has a similar form (3.1.2) as $x$. Let $e_{i}$ be the orthogonal projection of $K \otimes Y$ onto $K_{x(i)} \otimes Y$. Then $\left\{e_{i} ; 1 \leq i \leq 20\right\}$ are mutually orthogonal and

$$
x(i)\left(e_{i}^{\perp}(K \otimes Y)\right)=x(i)\left(K_{x(i)}^{\perp} \otimes Y\right) \subset K_{x(i)} \otimes Y=e_{i}(K \otimes Y) .
$$

Let $v \in K \otimes Y$ with $\|v\|=1$, then there exists $p(1 \leq p \leq 20)$ with $\left\|e_{p}(v)\right\|^{2} \leq$ $1 / 20$. Since $x(p)$ is selfadjoint, by [P: Lemma 4] we have

$$
|\langle x(p) v, v\rangle| \leq\|x\|\left\{\left\|e_{p} v\right\|^{2}+2\left\|e_{p} v\right\|\left\|e_{p}^{\perp} v\right\|\right\} .
$$

Hence

$$
|\langle\hat{x} v, v\rangle| \leq\left(\frac{19}{20}+\frac{1}{20}\left(\frac{1}{20}+2\left(\frac{1}{\sqrt{20}}\right)\right)\|x\| \leq \frac{39}{40}\|x\| .\right.
$$

It implies that $\|\hat{x}\| \leq(39 / 40)\|x\|$ because $\hat{x}$ is self adjoint. Again, $\hat{x}$ has the form (3.1.2). We iterate this method and for a given $\varepsilon>0$ we have

$$
\hat{\hat{x}} \in \operatorname{conv}\left\{((\alpha * \gamma) \otimes \beta)^{n}(x): n \in \mathbb{N}\right\}
$$

which satisfies that $\|\hat{\hat{x}}\| \leq \varepsilon\|x\|$. Since $\psi$ is $((\alpha * \gamma) \otimes \beta)$-invariant, $\psi(\hat{\hat{x}})=\psi(x)$. Hence $|\psi(x)|=|\psi(\hat{\hat{x}})| \leq \varepsilon\|x\|$. Since $\varepsilon$ is arbitrary, we have $\psi(x)=0$.

Corollary 3.3. Under the same conditions as in Theorem 3.1, $\psi$ is an $(\alpha * \gamma) \otimes \beta$ invariant state of $(A * C) \otimes B$ if and only if there exists $a \gamma \otimes \beta$ invariant state $\omega$ of $C \otimes B$ such that

$$
\psi=\omega \cdot F, \text { for } F=\left(E_{\phi} * i d_{C}\right) \otimes i d_{B} .
$$

Proof. Let $\psi$ be an $(\alpha * \gamma) \otimes \beta$ invariant state of $(A * C) \otimes B$, then the restriction $\omega$ of $\psi$ to $C \otimes B$ is a $\gamma \otimes \beta$ invariant state of $C \otimes B$ and by Theorem $3.1 \psi=\omega \cdot F$. Conversely, let $\omega$ be a $\gamma \otimes \beta$ invariant state of $C \otimes B$ and put $\psi=\omega \cdot F$. Then $\psi((\alpha * \gamma) \otimes \beta(c \otimes b))=\omega(F(\gamma(c) \otimes \beta(b)))=\omega(\gamma \otimes$ $\beta(c \otimes b))=\psi(c \otimes b)$ for $c \in C$ and $b \in B$. Let $x=\sum_{i=1}^{n} x_{i} \otimes b_{i}$ for $x_{i} \in S$ and $b_{i} \in B$. Then $\psi(x)=\sum_{i} \omega\left(F\left(x_{i} \otimes b_{i}\right)\right)=0$. Since $(\alpha * \gamma) \otimes \beta(x)=\sum_{i=1}^{n} \alpha * \gamma\left(x_{i}\right) \otimes$ $\beta\left(b_{i}\right)$ and $\alpha^{*} \gamma\left(x_{i}\right) \in S$, we have $\psi((\alpha * \gamma) \otimes(x))=0$. Hence $\psi=\psi \cdot(\alpha * \gamma) \otimes \beta$.

\section{§4. Entropy for Reduced Free Products and Tensor Products}

In this section we show relations between Sauvageot and Thouvenot entropy for the reduced free products and tensor products of automorphisms 
with the free shifts. To fix notations, we first recall the definition of entropy in [ST]. Let $A$ be a unital $C^{*}$-algebra with a state $\phi$. A coupling of $(A, \phi)$ is a pair $(\psi, B)$, where $B$ is an abelian $C^{*}$-algebra and $\psi$ is a state on the $C^{*}$-algebra $A \otimes B$ whose restriction to $A$ (identified with $A \otimes 1$ ) is $\phi$. We denote by $\mu$ the probability measure on $B$ obtained from the restriction of $\psi$ to $B$.

Let $\alpha$ be a $\phi$ invariant automorphism of $A$ and $(\psi, B)$ be a coupling of $(A, \phi)$. Let $\beta$ be an automorphism of $B$ such that $\psi$ is $\alpha \otimes \beta$-invariant. For a finite partition $\mathscr{P}$ of $B$, which consists of orthogonal projections $\left\{p_{t} \in B: 1 \leq i \leq n, \sum_{i} p_{i}=1\right\}$, put

$$
H_{\mu}(\mathscr{P})=\sum_{i=1}^{n}-\mu\left(p_{i}\right) \log \mu\left(p_{i}\right)
$$

and

$$
h^{\prime}(\psi, \mathscr{P})=\underline{\lim }_{n \rightarrow \infty} \frac{1}{n} H_{\mu}\left(\bigvee_{i=0}^{n-1} \beta^{-i}(\mathscr{P})\right)-H_{\mu}(\mathscr{P})-S\left(\left.\phi \otimes \mu\right|_{\mathscr{P}}|\psi|_{A \otimes \mathscr{P}}\right) .
$$

Here $S(\cdot \mid \cdot)$ is the relative entropy of states ([PW], [K]). Then Sauvageot and Thouvenot entropy is defined by

$$
H_{\phi}(\alpha)=\sup h^{\prime}(\psi, \mathscr{P}),
$$

where the sup is taken over all couplings $(\psi, B)$, partitions $\mathscr{P}$ and automorphisms $\beta$ as above.

From now, let $A_{0}$ be a unital $C^{*}$-algebra with a state $\phi_{0}$ and put $A_{i}=A_{0}$, $\phi_{i}=\phi_{0}$ for $i \in \mathbb{Z}$. Let $(A, \phi)=\left(* A_{i}, * \phi_{i}\right)_{i \in \mathbb{Z}}$.

Theorem 4.1. If $\alpha$ is the free shift on $A$, then

$$
H_{\phi * \rho}(\alpha * \gamma)=H_{\rho}(\gamma),
$$

where $\rho$ is a state on a unital $C^{*}$-algebra $C$ and $\gamma$ is a $\rho$-invariant automorphism on $C$.

Proof. Let $(\psi, B)$ be a coupling of $(A * C, \phi * \rho)$ such that $\psi$ is $(\alpha * \gamma) \otimes \beta$ invariant for an automorphism $\beta$ of $B$. We denote by $\psi_{1}$ the restriction of $\psi$ to $C \otimes B$ and by $\mu$ the restriction of $\psi$ to $1 \otimes B$. Let $F$ be the conditional expectation $\left(E_{\phi} * i d_{C}\right) \otimes i d_{B}$ as in Theorem 3.1. Then $(\phi * \rho) \otimes \mu=(\rho \otimes \mu) \cdot F$ and $\psi=\psi_{1} \cdot F$ by Theorem 3.1. Hence

$$
S((\phi * \rho) \otimes \mu \mid \psi)=S\left((\rho \otimes \mu) \cdot F \mid \psi_{1} \cdot F\right)=S\left(\rho \otimes \mu \mid \psi_{1}\right)
$$

by the invariance property of the relative entropy (see, $[\mathrm{CNT}: \mathbb{1},(7)])$. Taking sup $h^{\prime}(\cdot, \cdot)$, we have $H_{\phi * \rho}(\alpha * \gamma) \leq H_{\rho}(\gamma)$. Conversely let $B$ be a unital abelian $C^{*}$-algebra with a state $\mu$ and $\beta$ a $\mu$-invariant automorphism of $B$. If a 
$\gamma \otimes \beta$-invariant state $\psi^{\prime} \quad$ on $C \otimes B$ satisfies $\psi^{\prime}(c \otimes 1)=\rho(c), \quad(c \in C)$ and $\psi^{\prime}(1 \otimes b)=\mu(b),(b \in B)$, then $\psi^{\prime}$ is extended to an $(\alpha * \gamma) \otimes \beta$ invariant state $\psi$ on $(A * C) \otimes B$ by Corollary 3.3. The restriction of $\psi$ to $A * C$ (resp. B) is $\phi * \rho$ (resp. $\mu$ ). Hence we have $H_{\phi * \rho}(\alpha * \gamma) \geq H_{\rho}(\gamma)$ by the invariance property of the relative entropy. Therefore $H_{\phi * \rho}(\alpha * \gamma)=H_{\rho}(\gamma)$.

Remark 4.2. Let $C_{r}^{*}(G)$ be the reduced $C^{*}$-algebra of a discrete countable group $G$ and $\omega_{g}$ be the state on $C_{r}^{*}(G)$ defined by $\omega_{g}(\cdot)=\left\langle\cdot \delta_{g}, \delta_{g}\right\rangle$, where $\delta_{g}$ is the characteristic function of $g \in G$. We denote the identity of $G$ by e. Let $A_{0}=C_{r}^{*}(\mathbb{Z})$ and $\phi_{0}=\omega_{e}$. Put $A_{i}=A_{0}, \phi_{i}=\phi_{0}$ and $(A, \phi)=$ $\left(* A_{i}, * \phi_{i}\right)_{i \in \mathbb{Z}}$. Then $\left(C_{r}^{*}\left(F_{\infty}\right), \omega_{e}\right) \cong\left(C_{r}^{*}\left(F_{\infty}\right) * C_{r}^{*}(\mathbb{Z}), \phi * \phi_{0}\right) \cong\left(A * A_{0}, \phi * \phi_{0}\right)$, where $F_{\infty}$ is the free group on infinite generators. On a nuclear $C^{*}$ algebra, Sauvegeot-Thouvenot entropy coincides with Connes-Narnhofer-Thirring entropy, which coincides with Kolmogorov-Sinai entropy in the case of abelian algebras. Hence we have many values of Sauvageot-Thouvenot entropy for automorphisms on $C_{r}^{*}\left(F_{\infty}\right)$ by Theorem 4.1 .

Theorem 4.3. If $\alpha$ is the free shift on $A$, then

$$
H_{\phi \otimes \rho}(\alpha \otimes \gamma)=H_{\rho}(\gamma),
$$

where $\rho$ is a state of a unital $C^{*}$-algebra $C$ and $\gamma$ is a $\rho$-invariant automorphism on $C$.

Proof. Let $(\psi, B)$ be a coupling of $(A \otimes C, \phi \otimes \rho)$ such that $\psi$ is $\alpha \otimes \gamma \otimes \beta$ invariant for a automorphism $\beta$ of $B$. We denote by $\psi_{1}$ the state on $C \otimes B$ such that $\psi_{1}(y)=\psi(1 \otimes y)$ for an $y \in C \otimes B$ and by $F$ the conditional expectation $A \otimes C \otimes B$ onto $1 \otimes C \otimes B$ with $F(a \otimes y)=\phi(a) 1 \otimes y,(a \in A$, $y \in C \otimes B)$. Then $\phi \otimes \rho \otimes \mu=\left.(\phi \otimes \rho \otimes \mu)\right|_{1 \otimes C \otimes B} \cdot F$ and $\psi=\left.\psi\right|_{1 \otimes C \otimes B} \cdot F$ by Theorem 3.1. Hence

$$
\begin{aligned}
S(\phi \otimes \rho \otimes \mu \mid \psi) & =S\left(\left.(\phi \otimes \rho \otimes \mu)\right|_{1 \otimes C \otimes B} \cdot F|\psi|_{1 \otimes C \otimes B} \cdot F\right) \\
& =S\left(\left.(\phi \otimes \rho \otimes \mu)\right|_{1 \otimes C \otimes B}|\psi|_{1 \otimes C \otimes B}\right) \\
& =S\left(\rho \otimes \mu \mid \psi_{1}\right)
\end{aligned}
$$

by the invariance property. This implies $H_{\phi \otimes \rho}(\alpha \otimes \gamma) \leq H_{\rho}(\gamma)$. The converse inequality is trivial also by the invariance property of the relative entropy, because if a $\gamma \otimes \mu$-invariant state $\psi^{\prime}$ on $C \otimes B$ satisfies $\psi^{\prime}(c \otimes 1)=\rho(c),(c \in C)$ and $\psi^{\prime}(1 \otimes b)=\mu(b),(b \in B)$, then $\psi^{\prime}$ is extended to an $\alpha \otimes \gamma \otimes \beta$ invariant state $\psi$ on $A \otimes C \otimes B$ by $\psi^{\prime} \cdot E$ for the slice map (defined by $\phi$ ) $E$ of $A \otimes C \otimes B$ onto $C \otimes B$, and the restriction $\psi$ to $A \otimes C$ (resp. B) is $\phi \otimes \rho$ (resp. $\mu$ ). Hence we have the equality.

Remark. If we put $C=\mathbb{C} 1$ in Theorem 4.3, then we have [S: Remark 2].

Combining Theorem 4.1 and 4.3, we have: 
Corollary 4.4. If $\alpha$ is the free shift on $A$, then

$$
H_{\phi \otimes \rho}(\alpha \otimes \gamma)=H_{\rho}(\gamma)=H_{\phi * \rho}(\alpha * \gamma),
$$

where $\rho$ is a state of a unital $C^{*}$-algebra $C$ and $\gamma$ is a $\rho$-invariant automorphism on $C$.

\section{Acknowledgments}

This work was motivated by the talks of E. Størmer and D. Voiculescu in the workshop on Operator Algebra and Topology at Åarhus University in June of 1995. The author thanks U. Haagerup, J. Andersen and the members of GAP for inviting her to the workshop and their warm hospitalities. She also thanks E. Størmer for sending her his preprint [S] and the referee for pointing out the short proof of Cor. $2.2 \&$ kind linguistic corrections.

\section{References}

[A] Avitzour, D., Free products of $C^{*}$-algebras, Trans. Amer. Math. Soc., 271 (1982), 423-435.

[B] Boca, F., Free products of completely positive maps and spectral sets, J. Funct. Anal., 97 (1991), 251-263.

[CE] Choi, M. and Effros, E., Injectivity and operator spaces, J. Funct. Anal., 24 (1977), 156-209.

[CNT] Connes, A. Narnhofer, H. and Thirring, W., Dynamical entropy of $C^{*}$ algebras and von Neumann algebras, Comm. Math. Phys., 112 (1987), 691-719.

[K] Kosaki, H., Relative entropy of states: a variational expression, J. Operator Theory 16 (1986), 335-348.

[P] Popa, S., Markov traces on universal Jones algebras and subfactors of finite index, Invent. Math., 111 (1993), 375-405.

[P] Powers, S., Simplicity of the $C^{*}$-algebras associated with the free group in two generators, Duke Math. J., 42 (1975), 151-156.

[PW] Pusz, W. and Woronowicz, S., Form convex functions and the WYDL and other inequalities, Letter Math. Phys., 2 (1978), 505-512.

[ST] Sauvageot, J-L. and Thouvenot, J-P, Une nouvelle definition de l'entropie dynamique des systemes non commutatifs, Comm. Math. Phys., 145 (1992), 411-423.

[S] Størmer, E., States and shifts on infinite free products of $C^{*}$-algebras, Preprint in University of Oslo, 1995.

[V] Voiculescu, D., Symmetries of some reduced free product $C^{*}$-algebras, Operator Algebras and Their Connections with Topology and Ergodic Theory, Lecture notes in Math., Springer Verlag, 1132 (1985), 556-588.

[VDN] Voiculescu, D. Dykema, K. and Nica, A., Free randam variables, CRM Monograph series, Amer. Math. Soc., 1992.

Addendum. (March 16, 1996) F. Boca has kindly informed the author about his related work to Proposition 2.1: Completely positive maps on amalgamated product $C^{*}$-algebras, Math. Scand., 72 (1993), 212-222. 